\title{
Light detection: who needs eyes?
}

Kalyani Narasimhan

Nat. Neurosci. 1, 649 (1998)

Because of an editorial error, the affiliation for Russell Foster and colleagues was given incorrectly as University College London. The correct affiliation is Imperial College, University of London. We regret the error.

\section{Acetylcholine receptor M3 domain: stereochemical and volume contributions to channel gating}

Hai-Long Wang, Margherita Milone, Kinji Ohno, Xing-Ming Shen, Akira Tsujino, Anna Paola Batocchi, Pietro Tonali, Joan Brengman, Andrew G. Engel and Steven M. Sine

Nat. Neurosci. 2, 226-233 (1999)

On page 229, Fig. 4a-c reproduced poorly because of a printer's error. The correct version is below.

Kinetics of activation of receptors containing wild-type $\alpha$ (a), $\alpha$ V285I (b) or $\alpha$ V285A (c). Clusters of single-channel currents recorded at the indicated $\mathrm{ACh}$ concentrations from HEK cells expressing adult human nAChRs containing the indicated $\alpha$ subunits. Currents are shown at a bandwidth of $10 \mathrm{kHz}$, with channel openings as upward deflections.
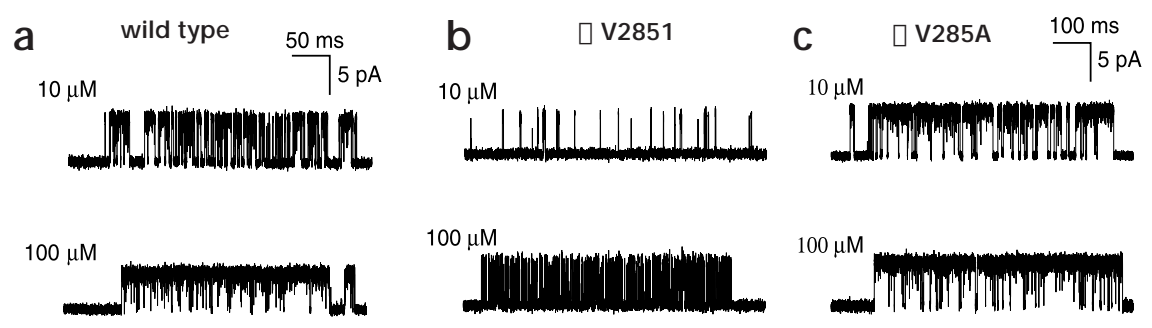

\section{Changes in cerebral cortex size are governed by fibroblast growth factor during embryogenesis}

Flora M. Vaccarino, Michael L. Schwartz, Rossana Raballo, Jon Nilsen, Julianne Rhee, Ming Zhou,

Thomas Doetschman, J. Douglas Coffin, Jason J. Wyland and Yu-Ting Elizabeth Hung

Nat. Neurosci. 2, 246-253 (1999)

Because of an editorial error, the correspondence address for this paper was omitted.

Correspondence should be addressed to F.M.V. (flora.vaccarino@yale.edu)

\section{Neuronal growth cone collapse triggers lateral extensions along trailing axons}

Roger W. Davenport, Edda Thies and Matthew L. Cohen

Nat. Neurosci. 2, 254-259 (1999)

On page 257, Fig. $4 \mathrm{~d}$ was printed without its red or green components because of a printer's error. The correct version is below.

(c) At the position of the lateral extension, two axons are seen continuing beyond the expanded region of the recently spread growth cone. (d) Serial sections containing axons were collected for electron microscopy, allowing tracing of individual axons. Fiduciary marks, predominantly organelles and large vesicles, aided in the determination of individual axons in multiple sections. The three axons are color coded, and tracings from each section are shown. The green and black axons were in the lead and were both induced to collapse. The red axon trailed, with its growth cone spread on the culture substratum after the lead growth cones collapsed. (e) Maximum number of axons that can be identified in a single section at any given position along the retinal ganglion cell fiber. Three individual axons are observed proximal to the lateral extension, and no more than two axons are observed distal to this point.
C

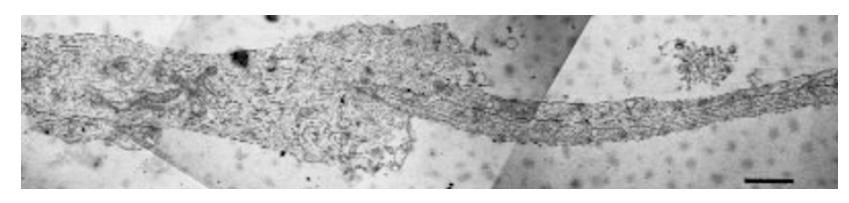

d

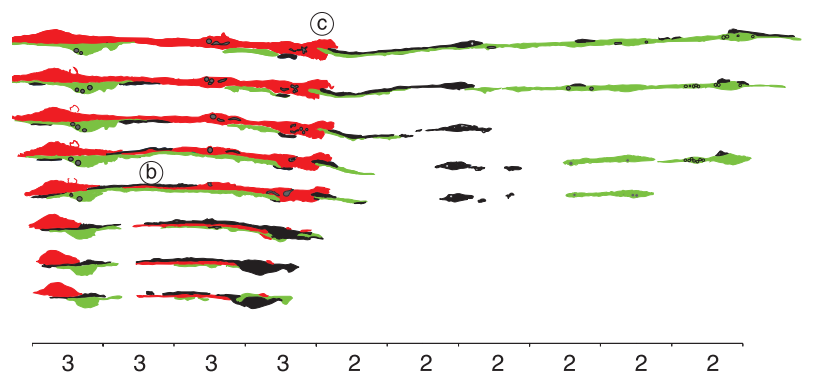

